Ottobre 2007

RR-07.64

Francesco Martinelli, Fabio Piedimonte

Optimal cycle production of a manufacturing system subject to deterioration 


\title{
Optimal cycle production of a manufacturing system subject to deterioration *
}

\author{
Francesco Martinelli and Fabio Piedimonte ${ }^{\dagger}$
}

\begin{abstract}
A single part type, single machine, fluid model manufacturing system is considered. The machine is subject to a deterministic deterioration process, which depends on the operation rate. The objective is to minimize a long term average cost index which penalizes inventory surplus and backlog. The optimal policy determined presents some similarities with the policy conjectured optimal for Markov failure prone systems. The considered problem can be applied to several real contexts, not only from the manufacturing domain, as briefly discussed in the paper.
\end{abstract}

\section{Introduction}

The problem of failure prone manufacturing systems has been investigated for a long time. Analytical results are known in some particular cases, e.g. if the failure process is Markov and the failure rate is an affine function of the production rate [4]. In this case the optimal control is the hedging point policy, according to which an optimal safety stock is reached as soon as possible (i.e. working at maximum capacity) and then maintained until the next failure. If the failure rate is a more general function of the production rate, the optimal control is not known and, based on a numerical investigation, the following conjecture was established [6]: if the failure rate is a concave function of the production rate, it seems optimal to follow a policy which differs from the hedging point policy only because the safety stock is maintained with an infinitely fast switching of the production rate between 0 and the maximum capacity. In the convex case, it seems optimal to smoothly decrease the production rate as the buffer level approaches the safety stock.

In order to gain insight into this problem, a slightly different version of it has been considered in this paper, where the down time is assumed deterministic and the uptime ends when the deterioration (which increases through a deterministic function of the production rate) reaches an alarm level. This has been done to simplify matters. Notice however that a deterministic formulation may be considered in several scenarios, like e.g. when the deterioration models a fuel consumption or a tool wearing process. Also, the repair time, in several situations, may be more accurately captured by a deterministic value rather than by a random exponentially distributed variable.

The deterministic formulation can be completely solved through the maximum principle. The optimal policy is as follows: if the deterioration rate is an affine function of the production rate, it is optimal to bring the buffer to 0 as soon as possible, maintain it at 0 working at constant rate and then, at the last possible time, increase the inventory toward an optimal safety stock working at maximum capacity. In the concave case the optimal policy differs from the one optimal in the affine case only because the buffer is maintained at 0 through an infinitely fast switching between 0 and the maximum capacity. In the convex case the production rate is smoothly modified as the buffer level increases. Even if this policy is different from the one conjectured optimal in the markovian case (due to the fact that the expected residual life in the Markovian case does not change over time unlike in the deterministic formulation where it is exactly known), many similarities can still be detected. In particular, in both the formulations, a major difference, with similar effects, arises between the convex and the concave case: in the affine and in the concave cases for example, both

\footnotetext{
*Work partially supported by MIUR under grants PRIN 2005092439

${ }^{\dagger}$ The authors are with Dipartimento di Informatica, Sistemi e Produzione, Università di Roma "Tor Vergata", via del Politecnico, 00133 Roma, Italy, e-mail: martinelli@disp.uniroma2.it.
} 
the formulations are solved by controls which increase the buffer only at maximum capacity. In addition, in the convex case, both the formulations bring to a control which is a smooth function of the buffer level while, in the concave case, an infinitely fast switching is adopted by the policies to keep the buffers constant. The similarity in the concave case is not surprising, since, intuitively, a concave dependence implies that intermediate production rates are optimally obtained through an infinitely fast switching between the maximum and the 0 production rate. Hence the optimal solution can be obtained from the affine solution by simply replacing intermediate production rates with switching productions. On the other hand, intermediate production rates, which may represent for the current buffer level the best tradeoff between buffer increment and deterioration (reliability in the Markov formulation), may be considered by the optimal policy when the deterioration rate (the failure rate in the Markov case) has a convex dependence on the production rate. It is then interesting to observe that in the affine case, in both the formulations, this tradeoff is obtained working at maximum capacity.

The generalization of these results to other failure models could be the subject of further investigations, even if this could require some additional assumptions on the failure/repair process.

It should be finally remarked that, in this paper, an optimal production planning, rather than an optimal maintenance problem (see e.g. [3]), is considered. The results in [7] are here extended by proving certain symmetry properties of the optimal solution and by providing a univocal relation between the convexity properties of the deterioration rate function and of the Hamiltonian evaluated along the optimal solution.

\section{Notation and problem formulation}

According to a standard notation, let $x(t)$ denote the buffer content at time $t$, with $x(t)>0$ representing an inventory surplus and $x(t)<0$ a backlog of $-x(t)$. Let $d$ be the constant demand rate to be met. Then the buffer level $x(t)$ at time $t$ satisfies the dynamical equation

$$
\dot{x}(t)=u(t)-d
$$

where the production rate $u(t) \equiv 0$ if at time $t$ the machine is in the down state (referred to as state 0 ), and $u(t) \in[0, \mu]$ if at time $t$ the machine is in the up state (referred to as state 1 ). The down time $T_{g}$ is constant and deterministic. The uptime is also deterministic but not constant: it depends on the production history since the last repair time $t_{0 f}$. In particular, we introduce

a deterioration function $z(t)=\int_{t_{0 f}}^{t}\left(a u^{\beta}(\tau)+b\right) d \tau$ where $a, b$ and $\beta$ are non-negative constants. The machine is stopped at time $t_{f f}$ where $t_{f f}$ is such that $z\left(t_{f f}\right)=1$. After a repair the machine is as good as new. This kind of deterioration function may be successfully employed to model tool wearing processes (see e.g. [9], [2] among others). In addition, notice that this choice allows to have a formulation similar to the Markov problem considered in the literature in the following sense: the uptime given by our deterministic problem if working at constant rate $\bar{u}$ is $1 /\left(a \bar{u}^{\beta}+b\right)$. This is also the expected uptime given in the Markov formulation [4] if working at the same constant rate $\bar{u}$.

An instantaneous quadratic cost $g[x(t)]=c x^{2}(t), c>0$, penalizing equivalently the backlog and the inventory surplus, is considered and the problem is to determine the control $u(\cdot)$ minimizing:

$$
J=\lim _{t \rightarrow+\infty} \frac{1}{t} \int_{t_{0}}^{t} c x^{2}(\tau) d \tau
$$

\subsection{An equivalent formulation}

Since the problem is deterministic and stationary, if an optimal control exists (this will be discussed below), the solution at steady state will be periodic. We will call cycle each period and $T$ its time duration. Let:

$$
J_{T}=\frac{1}{T} \int_{0}^{T} c x^{2}(t) d t
$$

Once the problem of minimizing (3) has been solved (such a minimization problem also includes the determination of the optimal initial condition $x(0)=X_{0}$ at the beginning of the cycle) with the 
constraint that the buffer level is the same at the beginning and at the end of the cycle, the original problem of minimizing (2) is simply solved by considering any policy which brings the buffer level to the optimal initial level $X_{0}$ and then, from that time on, applies the control minimizing (3) every $T$ time units. So, from now on, we will consider the problem of minimizing (3) with the constraint mentioned above that the buffer level is the same at the beginning and at the end of the cycle. Let $T_{f}$ be the uptime ( $T_{f}$ is a function of the production control law applied since the last failure, i.e. since 0 for the new problem). Since the machine is down in $\left[T_{f}, T\right]$, the problem of finding the optimal $u(\cdot)$ is restricted to $\left[0, T_{f}\right]$. Clearly $T=T_{f}+T_{g}$ is also a function of the control applied in $\left[0, T_{f}\right]$. Let $X_{0}$ and $X_{1}$ be respectively the buffer level at the beginning and at the end of the interval $\left[0, T_{f}\right]$. Then, considering the down interval $\left[T_{f}, T\right]$, the considered constraint is expressed by $X_{0}=X_{1}-d T_{g}$. Then, the index in (3) can be written taking into account the contributions in $\left[0, T_{f}\right]$ and in $\left[T_{f}, T\right]$. The first quantity depends on the production control, the second one, once $X_{1}$ has been computed, can be univocally determined:

$$
\frac{1}{T} \int_{T_{f}}^{T} c x^{2}(\tau) d \tau=\frac{c T_{g}\left(d^{2} T_{g}^{2}-3 X_{1} d T_{g}+3 X_{1}^{2}\right)}{3\left(T_{f}+T_{g}\right)} .
$$

To apply the maximum principle, an auxiliary variable $y(t):=\int_{0}^{t} c x^{2}(\tau) d \tau$ has been introduced, with $y_{f}:=y\left(T_{f}\right)$. The problem can be formulated as follows.

Problem 1 Determine the policy $u(\cdot)$ solving the following constrained optimization problem:

$$
\min _{u(\cdot)} \frac{y_{f}}{\left(T_{f}+T_{g}\right)}+\frac{c T_{g}\left(d^{2} T_{g}^{2}-3 X_{1} d T_{g}+3 X_{1}^{2}\right)}{3\left(T_{f}+T_{g}\right)}
$$

subject to

$$
[\dot{x}(t), \dot{z}(t), \dot{y}(t)]^{T}=\left[u(t)-d, a u^{\beta}(t)+b, c x^{2}(t)\right]^{T},
$$

$u(t) \in[0, \mu], 0 \leq t \leq T_{f}$, with the following boundary initial conditions:

$$
[x(0), z(0), y(0)]^{T}=\left[X_{1}-d T_{g}, 0,0\right]^{T}
$$

and final conditions:

$$
\left[x\left(T_{f}\right), z\left(T_{f}\right), y\left(T_{f}\right)\right]^{T}=\left[X_{1}, 1, y_{f}\right]^{T} .
$$

Let $\mathbf{x}(t):=[x(t), z(t), y(t)]^{T}$ be the complete state vector and $J^{*}$ the minimum of (4) (hence of $(3)$, and of (2) if the machine has enough capacity).

\section{$3 \quad$ Basic properties}

In this section, conditions under which it is possible to solve Problem 1, hence to obtain a finite $J$ in (2), are given together with some symmetry properties of the optimal solution.

\subsection{Feasibility analysis}

The system is said feasible if the machine has enough capacity to meet the demand. This corresponds to the possibility of finding a control law under which $J$ in $(2)$ is finite. Now, as mentioned above, an optimal control which guarantees that $J$ in (2) is finite will be periodic at steady state, hence it may exist if and only if the following equations can be satisfied:

$$
\left\{\begin{array}{l}
\int_{0}^{T_{f}}\left(a u^{\beta}(\tau)+b\right) d \tau=1 \\
\int_{0}^{T_{f}}(u(\tau)-d) d \tau=d T_{g} \\
u(t) \in[0, \mu] \quad 0 \leq t \leq T_{f}
\end{array}\right.
$$

To see if (8) can be solved we introduce the following feasibility function:

$$
S(u):=\frac{u-d}{a u^{\beta}+b}-d T_{g}
$$


Equation (9) represents the buffer level reached at the end of a cycle if the buffer level at time 0 starts at 0 and the machine (during the uptime) is operated at a constant production rate $u$. The following theorem, proved in [7], allows to analyze the feasibility problem in a straightforward manner.

Theorem 1 It is possible to find a solution to (8) (hence the system is feasible) if and only if there exists at least $a \bar{u} \in[0, \mu]$ such that $S(\bar{u}) \geq 0$.

Remark 1 Observe that the existence of $u$ such that $S(u)=0$ is enough to maintain the steady state periodic trajectory (i.e. to find a solution to (8)) and hence to have a finite $J$ in (2) but not, if there is no $u$ such that $S(u)>0$, to bring the buffer to any desired level. In this case the minimum of $J$ in (2) will be finite but dependent on initial conditions, hence larger, in general, than $J^{*}$.

\subsection{Symmetry properties of the optimal solution}

The optimal solution of Problem 1, during the up interval, satisfies certain symmetry properties, namely that the optimal control is a symmetric function of the time with respect to the time instant $T_{f} / 2$ while the corresponding optimal trajectory is anti-symmetric with respect to the same point on the time axis. This will help find the solution of Problem 1.

Theorem 2 [see Proof in the Appendix] For all $t \in\left[0, T_{f}\right]$, the optimal solution $\left(x^{*}(t), u^{*}(t)\right)$ of Problem 1 satisfies the following properties:

$$
\begin{aligned}
& x^{*}(t)=-x^{*}\left(T_{f}-t\right), \\
& u^{*}(t)=u^{*}\left(T_{f}-t\right) .
\end{aligned}
$$

Hence $X_{0}^{*}=-\frac{d T_{g}}{2}$ and $X_{1}^{*}=\frac{d T_{g}}{2}$.

\section{The optimal policy}

Due to the symmetry properties established above, $X_{1}$ is not a free variable, hence the cost in Problem 1 can be written as $\frac{y_{f}+K}{T_{f}+T_{g}}$, where the constant $K$ is specified below. This formulation with a final cost gives in this case more involved computations than its equivalent integral cost formulation. So, Problem 1 will be stated equivalently as follows (see details in Section 6.2):

Problem 2 Determine the policy $u(\cdot)$ solving the following constrained optimization problem:

$$
\min _{u(\cdot)} \int_{0}^{T_{f}} \frac{\left(t+T_{g}\right) c x^{2}(t)-y(t)-K}{\left(t+T_{g}\right)^{2}} d t
$$

where $K:=\frac{c d^{2} T_{g}^{3}}{12}$, subject to

$$
[\dot{x}(t), \dot{z}(t), \dot{y}(t)]^{T}=\left[u(t)-d, a u^{\beta}(t)+b, c x^{2}(t)\right]^{T} u(t) \in[0, \mu] \quad 0 \leq t \leq T_{f}
$$

with the following boundary initial conditions:

$$
[x(0), z(0), y(0)]^{T}=\left[-\frac{d T_{g}}{2}, 0,0\right]^{T}
$$

and final conditions:

$$
\left[x\left(T_{f}\right), z\left(T_{f}\right), y\left(T_{f}\right)\right]^{T}=\left[\frac{d T_{g}}{2}, 1, y\left(T_{f}\right)\right]^{T}
$$

The problem will be solved through the maximum principle (see [1]). Details on its application to this problem are given in the Appendix (see Section 6.3). 


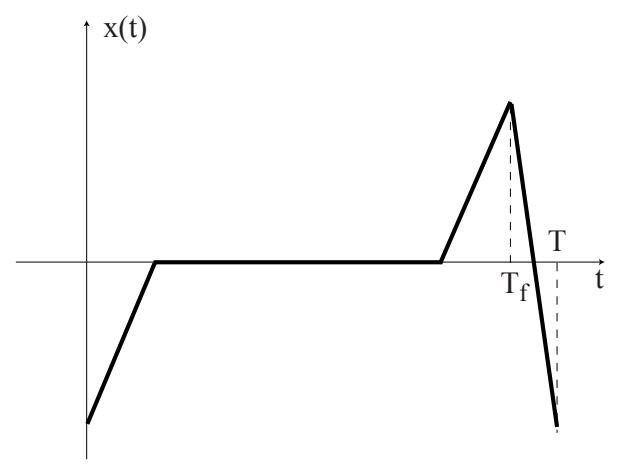

(a)

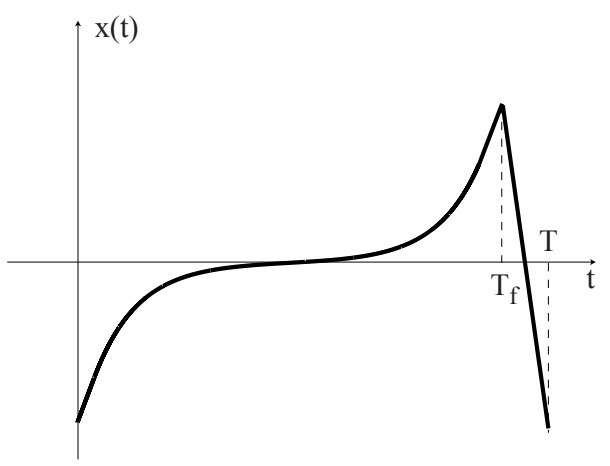

(b)

Figure 1: A qualitative plot of the $\mu d \mu$ and of the Smooth- $\mu d \mu$ Policies, optimal, respectively, in the affine $(\beta \in\{0,1\})$ and in the convex $(\beta>1)$ cases.

\subsection{The $\beta=0$ case}

This case is equivalent to the problem in [5] if the result found there is applied to a system with deterministic down times. If $\beta=0$, the final time

$$
T_{f}=\frac{1}{a+b}
$$

is independent of the control and the cost is minimized by the following policy.

Definition 1 ( $\mu d \mu$ Policy) The policy which operates the machine at maximum rate from $X_{0}$ to 0 , then maintains the buffer at 0 by working at rate $d$ and finally, at the last possible time, brings the buffer to $X_{1}$ through a maximum capacity production, will be denoted in the following $\mu d \mu$ (see Fig. 1(a)). Formally:

$$
u(t)= \begin{cases}\mu & 0 \leq t<\frac{d T_{g}}{2(\mu-d)} \\ d & \frac{d T_{g}}{2(\mu-d)} \leq t<T_{f}-\frac{d T_{g}}{2(\mu-d)} \\ \mu & T_{f}-\frac{d T_{g}}{2(\mu-d)} \leq t<T_{f}\end{cases}
$$

A direct computation shows that the $\mu d \mu$ policy is the only policy satisfying all the conditions of the maximum principle when $\beta=0$ (see Appendix - Section 6.4). Since if $\beta=0$ the Hamiltonian is jointly convex in the state and in the control, the maximum principle gives also sufficient conditions for optimality (see e.g. [8]). Hence the $\mu d \mu$ Policy is optimal in this case.

\subsection{The $\beta=1$ case}

If $\beta=1$, a reasoning similar to the one reported for the $\beta=0$ case can be followed to show the optimality of the $\mu d \mu$ Policy. Observe however that now the final time depends on the considered policy. In particular, the final time corresponding to the $\mu d \mu$ policy is now given by $T_{f}=\frac{1-a d T_{g}}{a d+b}$ (notice that $a d T_{g}<1$ if the system is feasible). Clearly, the first and the third phase of the $\mu d \mu$ policy have the same duration observed in the $\beta=0$ case (see (17)), but the duration of the second phase is different.

\subsection{The general case}

When $\beta \neq 0$ and $\beta \neq 1$ the problem becomes more involved. We will show in this section that the shape of the optimal solution strongly depends on the convexity properties of the deterioration rate function (i.e. on the value of $\beta$ ). 


\subsection{1 $\beta \in(0,1)$}

If $\beta \in(0,1)$, the cost index in Problem 2 is minimized by a policy called in the following $\mu \tilde{d} \mu$ policy, which is similar to the $\mu d \mu$ policy, but differs from it since the $d$-rate to maintain at 0 the buffer level will be obtained by an infinitely fast switching between rates 0 and $\mu$.

Definition 2 ( $\mu \tilde{d} \mu$ Policy) The $\mu \tilde{d} \mu$ is obtained from the $\mu d \mu$ Policy by maintaining the buffer level at 0 in the second phase with an infinitely fast switching between rates 0 and $\mu$, resulting in an average production rate $d$.

Remark 2 The $\mu \tilde{d} \mu$ policy is not suitable for real applications since an infinite switching frequency is not realizable. However, if $\beta \in(0,1)$, it is always possible to derive a finite value $N_{0}$ such that the cost corresponding to a policy which performs only a finite number $N \geq N_{0}$ of switches is smaller than the cost of the $\mu d \mu$ policy. If this $N_{0}$ is not too large for the specific real implementation, it could be used to reduce the cost of the $\mu d \mu$ policy.

We now prove that, when $\beta \in(0,1)$, the index in Problem 2 is minimized by the $\mu \tilde{d} \mu$ Policy. First we need the following lemma, proved in the Appendix, which states that, if $\beta \in(0,1)$, the optimal policy (if it exists) will never work at rates different from 0 and $\mu$.

Lemma 1 Assume $\beta \in(0,1)$ and let $u^{*}(t), t \in\left[0, T_{f}^{*}\right]$, be the optimal policy, if it exists. Then $u^{*}(t) \in\{0, \mu\}^{1}$ a.e. for all $t \in\left[0, T_{f}^{*}\right]$.

Theorem 3 If $\beta \in(0,1)$, the $\mu \tilde{d} \mu$ Policy solves Problem 2.

Proof. For Lemma 1, any rate different from 0 and $\mu$ will be (at least ideally) optimally substituted by an infinitely fast switching between 0 and $\mu$. But then, if we use this infinitely fast switching policy, the problem is as if the machine, whose deterioration rate function is $a u^{\beta}+b$, deteriorates according to a line through $(0, b)$ and $\left(\mu, a \mu^{\beta}+b\right)$, for which we know that the optimal policy is the $\mu d \mu$ Policy. So, if $\beta \in(0,1)$, just consider the $\mu d \mu$ Policy with the $d$ rate replaced by an infinitely fast switching as in the $\mu \tilde{d} \mu$ Policy.

Clearly the $\mu \tilde{d} \mu$ Policy only provides an asymptotic optimal limit, which can be considered in real contexts as mentioned in Remark 2.

\subsection{2 $\beta>1$}

If $\beta>1$, the optimal control can be derived through a numeric procedure. In fact, in this case, from equation (42) below (notice that, in view of Theorem 4 below, $\lambda_{2}^{*}>0$ ), the optimal control is given by:

$$
u^{*}(t)= \begin{cases}0 & \text { if } \lambda_{1}^{*}(t) \geq 0 \\ \min \left\{\mu,\left(-\frac{\lambda_{1}^{*}(t)}{a \beta \lambda_{2}^{*}}\right)^{\frac{1}{\beta-1}}\right\} & \text { if } \lambda_{1}^{*}(t)<0\end{cases}
$$

Now, following a reasoning similar to the one used in the application of the maximum principle in the $\beta=0$ case (see Appendix - Section 6.4), it is possible to show that, if an optimal policy exists, $\lambda_{1}^{*}(t)<0$ for all $t$. This implies from (18) that the optimal control will be:

$$
u^{*}(t)=\min \left\{\mu,\left(-\frac{\lambda_{1}^{*}(t)}{a \beta \lambda_{2}^{*}}\right)^{\frac{1}{\beta-1}}\right\},
$$

i.e., exploiting (41) below, of the type:

$$
u^{*}(t)=\min \left\{\mu,\left(C_{1}+C_{2} \int_{0}^{t} x(\tau) d \tau\right)^{\frac{1}{\beta-1}}\right\}
$$

where $C_{1}$ and $C_{2}$ are two positive constants. Given $C_{1}$, a unique $C_{2}$ may exist satisfying (8) with a $x(t)$ symmetric around $x\left(T_{f} / 2\right)=0$. So, the set of admissible values for $C_{1}$ and $C_{2}$ is actually a

\footnotetext{
${ }^{1}$ Notice that $\{0, \mu\}$ is not the interval $[0, \mu]$ but just the set consisting of the two elements 0 and $\mu$
} 
curve in the $\left(C_{1}, C_{2}\right)$ plane and we can consider, in this case, the cost $J$ in (2) as a function of $C_{1}$, i.e. $J=J\left(C_{1}\right)$. Then, the optimal policy may be derived by numerically finding the value of $C_{1}$ which minimizes $J\left(C_{1}\right)$. Observe that the set of $C_{1}$ for which a $C_{2}$ can be found to satisfy (8) with a $x(t)$ symmetric around $x\left(T_{f} / 2\right)=0$ is a bounded interval. A numeric search of the optimal $C_{1}$ will be then performed in this interval (see also [7])). In the remaining of the paper, this numeric search will be referred to as $C_{1}$-search and the policy generated by this procedure will be called Smooth $-\mu d \mu$, since in this case the production rate, starting from a value possibly smaller than $\mu$, is smoothly decreased as the buffer level approaches 0 , reaching a minimum value in general larger than $d$ when the buffer is cleared (see Fig. 1(b)).

Definition 3 (Smooth- $\mu d \mu$ Policy) The policy obtained from the $C_{1}$-search procedure mentioned above will be called Smooth $-\mu d \mu$ policy.

The numeric $C_{1}$-search procedure presented above is based on the fact that the Hamiltonian in (31), evaluated along the optimal policy, is convex in $u$. Then, to complete our investigations, we show that this is the case if $\beta>1$. We first need the following lemma, proved in the Appendix.

Lemma 2 The $\mu \tilde{d} \mu$ is the unique policy satisfying the maximum principle with a Hamiltonian strictly concave in $u$.

We can now state that if $\beta>1$ the Hamiltonian along the optimal trajectory is strictly convex in $u$. The following theorem is proved in the Appendix.

Theorem 4 Assume $\beta>1$. Then, if an optimal policy exists, it satisfies the maximum principle with a Hamiltonian strictly convex in $u$.

Since if $\beta>1$ the Hamiltonian in (31) is jointly convex with respect to the state and the control (just substitute (40) in (31) and use Theorem 4), also if $\beta>1$ the maximum principle provides sufficient conditions of optimality.

\subsubsection{Main result}

We can summarize all our investigations with the following theorem.

Theorem 5 The solution of Problem 1, if the system is feasible, is:

- the $\mu d \mu$ Policy if $\beta=0$ or $\beta=1$;

- the Smooth $-\mu d \mu$ Policy if $\beta>1$.

If $\beta \in(0,1)$, the $\mu \tilde{d} \mu$ Policy gives an asymptotic solution for Problem 1.

Numerical examples of the results presented in this paper can be found in [7].

\section{Conclusions}

An optimal backlog/inventory problem for a machine characterized by a deterministic deterioration process depending on the working rate has been considered and solved. The obtained solution presents a significant analogy with the control conjectured optimal for a similar Markov formulation of the problem.

The result obtained, which could be possibly exploited to investigate situations where the uptimes obey to other probability distributions, is the following one: when the deterioration rate of the machine has a concave dependence on the working rate, the optimal policy never works at rates different from 0 or the maximum allowed capacity. On the contrary, in the convex case, these intermediate rates, which may represent the best tradeoff between buffer increment and deterioration rate, could be used by the optimal policy.

To fix ideas a manufacturing system has been considered (also for comparison purposes with the Markov formulation), but the model can be applied to all situations where a device is characterized by a deterioration process (or fuel/energy consumption) which depends on the working rate. 


\section{Appendix}

\subsection{Proof of Theorem 2}

Consider the following change of variables:

$$
\begin{aligned}
\tau & :=T_{f}-t \\
\chi(\tau) & :=-x\left(T_{f}-\tau\right), \\
v(\tau) & :=u\left(T_{f}-\tau\right) \\
\theta(\tau) & :=y\left(T_{f}\right)-y\left(T_{f}-\tau\right) \\
\zeta(\tau) & :=1-z\left(T_{f}-\tau\right) \\
\chi_{1} & :=d T_{g}-X_{1}
\end{aligned}
$$

and write Problem 1 with the new variables. It is easy to check that we get the same formulation with the new variables, in particular, with the considered substitution, we obtain:

$$
\begin{gathered}
\frac{y_{f}}{\left(T_{f}+T_{g}\right)}+\frac{c T_{g}\left(d^{2} T_{g}^{2}-3 X_{1} d T_{g}+3 X_{1}^{2}\right)}{3\left(T_{f}+T_{g}\right)} \rightarrow \\
\rightarrow \frac{\theta_{f}}{\left(T_{f}+T_{g}\right)}+\frac{c T_{g}\left(d^{2} T_{g}^{2}-3 \chi_{1} d T_{g}+3 \chi_{1}^{2}\right)}{3\left(T_{f}+T_{g}\right)}, \\
{[\dot{\chi}(\tau), \dot{\zeta}(\tau), \dot{\theta}(\tau)]^{T}=\left[v(\tau)-d, a v^{\beta}(\tau)+b, c \chi^{2}(\tau)\right]^{T},}
\end{gathered}
$$

and

$$
\begin{gathered}
{[\chi(0), \zeta(0), \theta(0)]^{T}=\left[\chi_{1}-d T_{g}, 0,0\right]^{T},} \\
{\left[\chi\left(T_{f}\right), \zeta\left(T_{f}\right), \theta\left(T_{f}\right)\right]^{T}=\left[\chi_{1}, 1, \theta_{f}\right]^{T}}
\end{gathered}
$$

Since the problem with the new variables is like the problem with the old variables, also the optimal solution in the new variables will be as the optimal solution in the old variables, i.e.

$$
\begin{aligned}
& \chi^{*}(\delta)=x^{*}(\delta), \\
& v^{*}(\delta)=u^{*}(\delta),
\end{aligned}
$$

for all $\delta \in\left[0, T_{f}\right]$. From (27)-(28) and (22)-(23), it is possible to see that:

$$
\begin{aligned}
& x^{*}(t)=\chi^{*}(t)=-x^{*}\left(T_{f}-t\right), \\
& u^{*}(t)=v^{*}(t)=u^{*}\left(T_{f}-t\right) .
\end{aligned}
$$

Based on the symmetry properties of $x^{*}(\cdot)$, we have $x^{*}\left(T_{f}\right)=-x^{*}(0)$, hence:

$$
X_{1}^{*}=x^{*}\left(T_{f}\right)=-x^{*}(0)=-\left(X_{1}^{*}-d T_{g}\right)
$$

from which the value of $X_{1}^{*}$ (and $X_{0}^{*}=x^{*}(0)$ ) can be immediately derived.

\subsection{Formulation with integral cost}

The original formulation of Problem 1 only includes a final cost which can be written as follows: $F\left(T_{f}\right)=\left.\frac{y(t)+K}{t+T_{g}}\right|_{t=T_{f}}$, where $K=\frac{c d^{2} T_{g}^{3}}{12}$ is a constant (its value has been computed taking into account that $\left.X_{1}^{*}=\frac{d T_{g}}{2}\right)$. Clearly, this final cost can be written in an integral form: $F\left(T_{f}\right)=$ $F(0)+\int_{0}^{T_{f}} f(t) d t$ where $f(t)=\frac{d F}{d t}$. This allows to obtain the integral cost expression (12) in Problem 2, since $F(0)=-\frac{K}{T_{g}}$ does not give any contribution to the shape of the optimal policy (but only on the value of the optimal cost). 


\subsection{The application of the maximum principle to Problem 2}

Using the maximum principle (see e.g. [1]), the Hamiltonian for Problem 2 is given by (omitting for brevity the dependence of $\lambda_{1}, \lambda_{2}, \lambda_{3}$ on the time):

$$
H=\frac{\left(t+T_{g}\right) c x^{2}(t)-y(t)-K}{\left(t+T_{g}\right)^{2}}+\lambda_{1}[u(t)-d]+\lambda_{2}\left[a u^{\beta}(t)+b\right]+\lambda_{3} c x^{2}(t)
$$

The auxiliary system is:

$$
\begin{aligned}
\frac{d \lambda_{1}(t)}{d t} & =-\frac{\partial H(\mathbf{x}(t), u(t), t, \lambda(t))}{\partial x}=-\left[\frac{1}{t+T_{g}}+\lambda_{3}(t)\right] 2 c x(t) \\
\frac{d \lambda_{2}(t)}{d t} & =-\frac{\partial H(\mathbf{x}(t), u(t), t, \lambda(t))}{\partial z}=0 \\
\frac{d \lambda_{3}(t)}{d t} & =-\frac{\partial H(\mathbf{x}(t), u(t), t, \lambda(t))}{\partial y}=\frac{1}{\left(t+T_{g}\right)^{2}}
\end{aligned}
$$

If a policy $u^{*}(t)$ is optimal, then there exist $\lambda_{1}^{*}(t), \lambda_{2}^{*}(t)$ and $\lambda_{3}^{*}(t)$, such that this control function and the corresponding optimal trajectory are such that:

$$
\begin{gathered}
u^{*}(t)=\arg \min _{u \in[0, \mu]}\left[\lambda_{1}^{*}(t) u+\lambda_{2}^{*}(t) a u^{\beta}\right] \\
H^{*}(t)=-\int_{t}^{T_{f}}\left[-\frac{c x^{* 2}(\tau)}{\left(\tau+T_{g}\right)^{2}}+\frac{2\left(y^{*}(\tau)+K\right)}{\left(\tau+T_{g}\right)^{3}}\right] d \tau \\
0=H^{*}\left(T_{f}^{*}\right)=\frac{\left(T_{f}^{*}+T_{g}\right) c x^{* 2}\left(T_{f}^{*}\right)-y^{*}\left(T_{f}^{*}\right)-K}{\left(T_{f}^{*}+T_{g}\right)^{2}}+\lambda_{1}^{*}\left(T_{f}^{*}\right)\left[u^{*}\left(T_{f}^{*}\right)-d\right]+ \\
+\lambda_{2}^{*}\left(T_{f}^{*}\right)\left[a u^{* \beta}\left(T_{f}^{*}\right)+b\right]+\lambda_{3}^{*}\left(T_{f}^{*}\right) c x^{* 2}\left(T_{f}^{*}\right) \\
\lambda_{3}^{*}\left(T_{f}^{*}\right)=0,
\end{gathered}
$$

where (37) and (38) are respectively the transversality and the orthogonality conditions. From (33):

$$
\lambda_{2}^{*}(t)=\lambda_{2}^{*} .
$$

Through the integration of (34), using (38), we get:

$$
\lambda_{3}^{*}(t)=\frac{1}{T_{f}^{*}+T_{g}}-\frac{1}{t+T_{g}}
$$

Substituting (40) in (32):

$$
\frac{d \lambda_{1}^{*}(t)}{d t}=-\frac{2 c}{T_{f}^{*}+T_{g}} x^{*}(t)
$$

Now, let $u^{*}(t) \in[0, \mu]$ be an admissible control which drives the system from an admissible initial state to an admissible final state (i.e. which satisfy (14) and (15)). Let $\mathbf{x}^{*}(t)$ be the state trajectory (13) corresponding to $u^{*}(t)$. Then, if $u^{*}(t)$ is an optimal solution for Problem 2, there exist a function $\lambda_{1}^{*}(t)$ and a constant $\lambda_{2}^{*}$ such that (41) holds and, in addition:

$$
\begin{gathered}
u^{*}(t)=\arg \min _{u \in[0, \mu]}\left[\lambda_{1}^{*}(t) u+\lambda_{2}^{*} a u^{\beta}\right] \\
-\frac{y^{*}(t)+K}{\left(t+T_{g}\right)^{2}}+\lambda_{1}^{*}(t)\left[u^{*}(t)-d\right]+\lambda_{2}^{*}\left[a u^{* \beta}(t)+b\right]+\frac{1}{T_{f}^{*}+T_{g}} c x^{* 2}(t)= \\
=-\int_{t}^{T_{f}}\left[-\frac{c x^{* 2}(\tau)}{\left(\tau+T_{g}\right)^{2}}+\frac{2\left(y^{*}(\tau)+K\right)}{\left(\tau+T_{g}\right)^{3}}\right] d \tau \\
\frac{\left(T_{f}^{*}+T_{g}\right) c d^{2} T_{g}^{2}-4\left(y^{*}\left(T_{f}^{*}\right)+K\right)}{4\left(T_{f}^{*}+T_{g}\right)^{2}}+\lambda_{1}^{*}\left(T_{f}^{*}\right)\left[u^{*}\left(T_{f}^{*}\right)-d\right]+\lambda_{2}^{*}\left[a u^{* \beta}\left(T_{f}^{*}\right)+b\right]=0
\end{gathered}
$$




\subsection{The $\mu d \mu$ Policy is the unique policy satisfying the maximum princi- ple when $\beta=0$}

By substituting the buffer trajectory $x(t)$ resulting from the application of (17) in (41), it is possible to derive $\lambda_{1}(t)$ as a function of the initial condition $\lambda_{10}$. The $\mu d \mu$ policy consists of three phases, the first and the third characterized by a production rate $\mu$ and the second one by a production rate $d$. In both the phases where the production rate is $\mu, \lambda_{1}(t)$ is quadratic and concave. In the second phase, $\lambda_{1}(t)$ is constant. Observe that $\lambda_{1}(t)$ is continuous for all $t$ and is symmetric with respect to $T_{f} / 2$. We compute $\lambda_{10}$ in such a way that $\lambda_{1}(t)=0$ in the second phase, hence it will be negative in the other two phases. Let $\lambda_{10}^{*}$ be the value of $\lambda_{10}$ that guarantees this property (clearly $\lambda_{10}^{*}<0$ ) and let $\lambda_{1}^{*}(t)$ be the corresponding $\lambda_{1}(t)$. From (44), using the symmetry (i.e. $\left.\lambda_{1}^{*}\left(T_{f}\right)=\lambda_{10}^{*}\right)$ it is possible to compute $\lambda_{2}^{*}$ :

$$
\lambda_{2}^{*}=\frac{c d^{2} \mu T_{g}^{3}(a+b)}{12(\mu-d)\left(a T_{g}+b T_{g}+1\right)^{2}}
$$

which is clearly positive. A direct computation allows to show that (43) is satisfied. As for (42), we have:

$$
u(t)=\arg \min _{u \in[0, \mu]}\left[\lambda_{1}(t) u+\lambda_{2} a\right]
$$

Now, $\lambda_{1}(t) u+\lambda_{2} a$ is a line with slope $\lambda_{1}(t)$ : when $\lambda_{1}(t)<0$, the minimum is achieved by $u=\mu$; when $\lambda_{1}(t)>0$, the minimum is achieved by $u=0$; when $\lambda_{1}(t)=0,(46)$ is independent of $u$ and it is then possible to work at any rate between 0 and $\mu$. Now, the considered $\lambda_{1}^{*}(t)$ and the $\mu d \mu$ policy minimize (46) since $u=\mu$ in the first and in the third phases where $\lambda_{1}^{*}(t)$ is negative, and $u=d$ in the second phase where $\lambda_{1}^{*}(t)=0$. So the $\mu d \mu$ policy satisfies the maximum principle.

On the other hand, it is possible to see that there is no other policy satisfying the maximum principle. At the beginning $x(t)$ is negative, hence, for $(41), \lambda_{1}(t)$ is increasing. If $\lambda_{10}$ were larger than or equal to $0, u(t)$ would be equal to 0 for $(46)$, hence $x(t)$ would decrease for ever, since $\lambda_{1}(t)$ would be always positive and increasing. Similarly, if $\lambda_{10}^{*}<\lambda_{10}<0, \lambda_{1}(t)$ would become 0 when $x(t)$ is still negative and, for (41), will continue to increase reaching positive values. Then $u(t)$ would become 0 and $x(t)$ would start to decrease for ever as in the previous case. Finally, if $\lambda_{10}<\lambda_{10}^{*}, \lambda_{1}(t)$ would be always negative and $x(t)$ would increase for ever, reaching and passing the maximum value $X_{1}$. So, it is necessary that $\lambda_{1}(t)$ becomes 0 when $x(t)$ becomes 0 . At that moment it is possible to select $u(t)=d$ in such a way that $x(t)$ and $\lambda_{1}(t)$ remain at 0 . If at some time one selects $u(t)<d, x(t)$ becomes negative, $\lambda_{1}(t)$ becomes positive implying $u(t)=0$ and $x(t)$ would decrease for ever. Viceversa, if at some time one selects $u(t)>d, x(t)$ becomes positive, $\lambda_{1}(t)$ becomes negative implying $u(t)=\mu$ and $x(t)$ would increase for ever. In both the cases, we would end up with a final value different from $X_{1}$ (smaller in the first case, larger in the second case).

\subsection{Proof of Lemma 1}

The trajectories defined in this proof are represented in Fig. 2. Assume by contradiction that there exists a finite interval $I=\left[t_{a}, t_{b}\right] \subseteq\left[0, T_{f}^{*}\right], t_{b}-t_{a}>0$, such that for all $t \in I, u^{*}(t) \notin\{0, \mu\}$ a.e. and construct a new policy $\hat{u}(\cdot)$ by substituting the production rate of the assumed optimal policy in $I$ with a fast switching between 0 and $\mu$. This would provide (thanks to the concavity of the deterioration rate function) a lower deterioration of the machine when working with the new policy $\hat{u}(\cdot)$. The extra working time gained could then be used to maintain the buffer at 0 . This would give the same integral $\int_{0}^{T_{f}} c x^{2}(t) d t$ but a larger $T_{f}$, so a smaller cost $J$.

To be more specific, let $T_{i}=t_{b}-t_{a}$ be the duration of the considered time interval $I$ where $u^{*}(t) \notin\{0, \mu\}$ and let $\Delta t=T_{i} / N$, with $N$ a large integer specified in the following. Consider a partition of $I$ with $t_{k}=t_{a}+k \Delta t, k=0,1, \ldots N$ (hence $t_{0} \equiv t_{a}$ and $t_{N} \equiv t_{b}$ ). Let $u_{k}^{*}$ be the average production rate of the optimal policy in $\left[t_{k}, t_{k+1}\right]$. We have $u_{k}^{*} \notin\{0, \mu\}$. Then consider a policy $\tilde{u}_{N}(\cdot)$ as follows: $\tilde{u}_{N}(t)=u^{*}(t)$ for all $t \in\left[0, t_{a}\right]$ and for all $t \in\left[t_{b}, T_{f}^{*}\right]$. Then, for all $k=0, \ldots, N-1$, take $\tilde{u}_{N}(t)=\mu$ for all $t \in\left[t_{k}, t_{k}+\alpha_{k} \Delta t\right]$ and $\tilde{u}_{N}(t)=0$ for all $t \in\left[t_{k}+\alpha_{k} \Delta t, t_{k+1}\right]$, where $\alpha_{k} \in(0,1)$ is such that the average production rate of $\tilde{u}_{N}(\cdot)$ in $\left[t_{k}, t_{k+1}\right]$ is as the average 


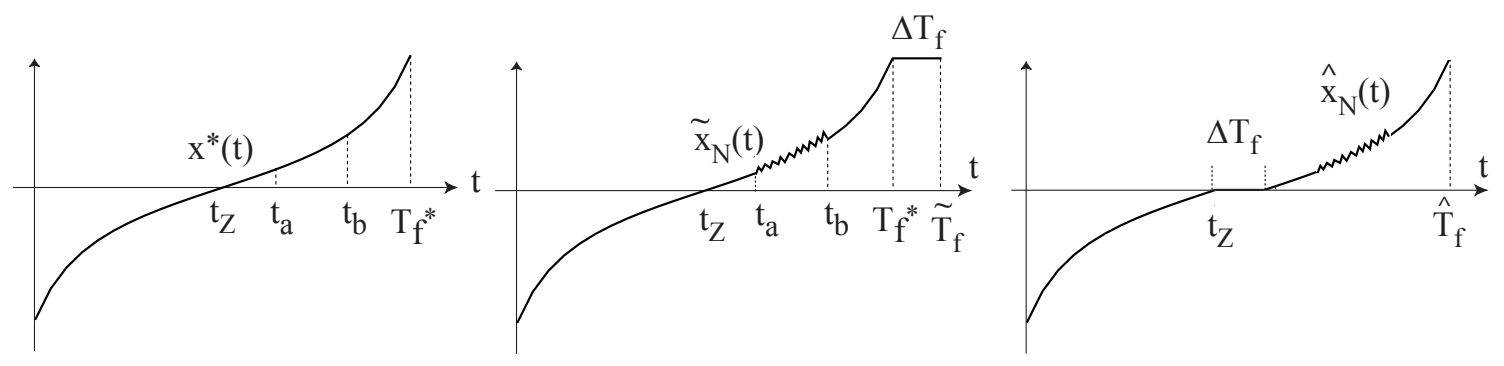

Figure 2: Trajectories in the proof of Lemma 1.

production rate of $u^{*}(\cdot)$ in the same interval, i.e. $\alpha_{k} \mu=u_{k}^{*}$. In this way, if $x^{*}(\cdot)$ and $\tilde{x}_{N}(\cdot)$ are the trajectories corresponding to the assumed optimal policy $u^{*}(\cdot)$ and to the new policy $\tilde{u}_{N}(\cdot)$ respectively, we have $x^{*}\left(t_{k}\right) \equiv \tilde{x}_{N}\left(t_{k}\right)$ for all $k=0,1, \ldots, N$. But clearly, due to the concavity of the deterioration rate function, for any $N$ selected (included the limit as $N \rightarrow \infty$ ), the deterioration in the interval $I$ corresponding to $\tilde{u}_{N}(\cdot)$ is independent of $N$ and is strictly less than the deterioration in the same interval corresponding to the optimal policy. To see this, let $u_{\text {avg }}$ be the average production rate corresponding to $u^{*}(\cdot)$ in I. For all $N$ (included $N \rightarrow \infty$ ), $u_{\text {avg }}$ is also the average production rate in $I$ of $\tilde{u}_{N}(\cdot)$. Let now $L_{1}(u)$ be the line through the points $(0, b)$ and $\left(\mu, a \mu^{\beta}+b\right)$ and $L_{2}(u)$ be the line through the points $\left(u_{\min }, a u_{\min }^{\beta}+b\right)$ and $\left(u_{\max }, a u_{\max }^{\beta}+b\right)$, where $u_{\min }:=\min _{t \in I} u^{*}(t)>0$ and $u_{\max }:=\max _{t \in I} u^{*}(t)<\mu$. Then we have (for all $\left.N\right)$ :

$$
z^{*}\left(t_{b}\right)-z^{*}\left(t_{a}\right) \geq T_{i} L_{2}\left(u_{\text {avg }}\right)>T_{i} L_{1}\left(u_{\text {avg }}\right) \equiv \tilde{z}_{N}\left(t_{b}\right)-\tilde{z}_{N}\left(t_{a}\right)
$$

with the same convention on $z$ adopted for $x$. This implies that $\tilde{z}_{N}\left(T_{f}^{*}\right)<z^{*}\left(T_{f}^{*}\right)=1$ for any $N$, included $N \rightarrow \infty$. So, using $\tilde{u}_{N}(\cdot)$, with any $N$, the machine can work up to a time $\tilde{T}_{f}>T_{f}^{*}$, which, as shown above, is independent of $N$. We can use the extra time $\tilde{T}_{f}-T_{f}^{*}$ to maintain the buffer at 0 . Denote by $\Delta T_{f}$ the extra time $\tilde{T}_{f}-T_{f}^{*}$ when the machine during the extra time available is operated at rate $d$. So $\Delta T_{f}$ is such that $\tilde{z}_{N}\left(T_{f}^{*}\right)+\Delta T_{f}\left(a d^{\beta}+b\right)=1$.

Now, let $t_{Z} \in\left(0, T_{f}^{*}\right)$ be the first time such that $x^{*}\left(t_{Z}\right)=0$ and assume without loss of generality (due to Theorem 2) that $t_{Z}<t_{a}$. So also $\tilde{x}_{N}\left(t_{Z}\right)=0$. Then set $\hat{u}_{N}(t) \equiv \tilde{u}_{N}(t)$ for all $t<t_{Z}, \hat{u}_{N}(t)=d$ for all $t \in\left[t_{Z}, t_{Z}+\Delta T_{f}\right], \hat{u}_{N}(t) \equiv \tilde{u}_{N}\left(t-\Delta T_{f}\right)$ for all $t \in\left(t_{Z}+\Delta T_{f}, \hat{T}_{f}\right)$, where $\hat{T}_{f} \equiv \tilde{T}_{f}$ is such that $\hat{z}_{N}\left(\hat{T}_{f}\right)=1$. It easy to check that $\hat{x}_{N}\left(\hat{T}_{f}\right) \equiv x^{*}\left(T_{f}^{*}\right)$ for any $N$ selected. Now, let

$$
\hat{c}_{N}:=\int_{0}^{\hat{T}_{f}} c\left[\hat{x}_{N}(t)\right]^{2} d t, \quad c^{*}:=\int_{0}^{T_{f}^{*}} c\left[x^{*}(t)\right]^{2} d t .
$$

Then, since $\lim _{N \rightarrow \infty} \hat{c}_{N}=c^{*}$, for all $\epsilon$ it is possible to find $\bar{N}$ such that, for all $N>\bar{N}, \hat{c}_{N}<c^{*}+\epsilon$. Consider a value $0<\epsilon_{0}<\Delta T_{f}$ (hence $\hat{T}_{f}=T_{f}^{*}+\Delta T_{f}>T_{f}^{*}+\epsilon_{0}$ ) and let $\bar{N}_{0}$ be such that $\hat{c}_{N}<c^{*}+\bar{\epsilon}_{0}$ for all $N>\bar{N}_{0}$, where $\bar{\epsilon}_{0}=\epsilon_{0} c^{*} / T_{f}^{*}$. Then for all $N>\bar{N}_{0}$, we have:

$$
\hat{J}_{N}-J^{*}=\frac{\hat{c}_{N}}{\hat{T}_{f}}-\frac{c^{*}}{T_{f}^{*}}<\frac{c^{*}+\bar{\epsilon}_{0}}{T_{f}^{*}+\epsilon_{0}}-\frac{c^{*}}{T_{f}^{*}} \equiv 0 .
$$

\subsection{Proof of Lemma 2}

Proceeding as for the $\beta=0$ case, the initial value $\lambda_{10}$ of $\lambda_{1}(t)$ must be selected in such a way that during the second (oscillating) phase the concave Hamiltonian is minimized at $u=0$ and at $u=\mu$, i.e. $\left.\left.H\right|_{u=0} \equiv H\right|_{u=\mu}$ thanks to the concavity assumption. This corresponds to: $\lambda_{1}(t)=-\lambda_{2} a \mu^{(\beta-1)}$. From (44):

$$
\lambda_{2}^{*}=\frac{c d^{2} T_{g}^{3}\left(a \mu^{\beta} d+b \mu\right)}{12\left(1+b T_{g}\right)^{2}(\mu-d)}
$$

which, like in the previous cases, is positive. It is possible to verify that also Equation (43) is fulfilled. In particular it is interesting to observe that, during the oscillation, (43) is satisfied by 
$u=0$ and by $u=\mu$ (and not by $u=d$ ). In [7] we showed how this policy satisfies also (41) and (42). So all the conditions of the maximum principle are satisfied if the Hamiltonian is strictly concave. It is the unique policy which satisfies the maximum principle: this can be verified exactly as done for the $\beta=0$ case.

\subsection{Proof of Theorem 4}

If $\beta>1$, for Lemma 2, the Hamiltonian on the optimal trajectory can not be strictly concave: this would imply that the maximum principle is satisfied only by the $\mu \tilde{d} \mu$ Policy, with a positive $\lambda_{2}^{*}$, hence with $\beta \in(0,1)$. So, assume that the Hamiltonian is affine (i.e. $\lambda_{2}^{*}=0$ ): in this case the only policy satisfying the maximum principle is the $\mu d \mu$, again with a $\lambda_{2}^{*}>0$. So the only possibility is that the Hamiltonian is strictly convex, i.e. $\lambda_{2}^{*}>0$.

\section{References}

[1] M. Athans and P. L. Falb, Optimal control: An introduction to the theory and its applications, McGraw-Hill, 1966.

[2] T. J. Drozda and C. Wick, Eds., Tools and Manufacturing Engineering Handbook, Vol. I, Dearbom, MI: Society Manufac. Eng., 1983.

[3] M. M. Hosseini, R. M. Kerr and R. B. Randall, "An Inspection Model with Minimal and Major Maintenance for a System with Deterioration and Poisson Failures," IEEE Transactions on Reliability, vol. 49, no. 1, pp. 88-98, 2000.

[4] J. Q. Hu, P. Vakili, and G. X. Yu, "Optimality of hedging point policies in the production control of failure-prone manufacturing systems," IEEE Transactions on Automatic Control, vol. AC-39, pp. 1875-1880, 1994.

[5] J. Q. Hu and D. Xiang, "Optimal control for systems with deterministic production cycles," IEEE Transactions on Automatic Control, vol. AC-40, pp. 782-786, 1995.

[6] G. Liberopoulos and M. Caramanis, "Production control of manufacturing systems with production-rate dependent failure rates," IEEE Transactions on Automatic Control, vol. AC39, no. 4, pp. 889-895, 1994.

[7] F. Martinelli and F. Piedimonte, "Production control of a manufacturing system subject to deterioration," in IEEE Conference on Decision and Control CDC 2005, Seville (Spain), December 2005.

[8] S. P. Sethi and G. L. Thompson, Optimal control theory: Applications to Management Science and Economics, Springer, 2000.

[9] Y.-C. Yen, J. Sohner, B. Lilly and T. Altan, "Estimation of tool wear in orthogonal cutting using the finite element analysis," Journal of Materials Processing Technology, vol. 146, pp. 8291, 2004. 